\title{
Mesoscopic thermal transport through a weak link
}

\author{
Michael R. Geller and Kelly R. Patton \\ Department of Physics and Astronomy, University of Georgia, Athens, Georgia 30602-2451
}

(July 8, 2001)

\begin{abstract}
We consider mesoscopic thermal transport between two bulk dielectrics joined by a narrow wire or weak mechanical link. In the "tunneling" regime where the phonon transmission probability through the link is small and the thermal conductance is much less than $\pi k_{\mathrm{B}}^{2} T / 6 \hbar$, the thermal current is determined by a product of the local vibrational spectral densities of the two bodies. We derive an expression for the thermal current that is a thermal analog of the well-known formula for the electrical current through a tunneling barrier.
\end{abstract}

PACS: $63.22 .+\mathrm{m}, 66.70 .+\mathrm{f}, 68.65 .-\mathrm{k}$

\section{INTRODUCTION}

Recently there has been considerable interest in the physics of phonons in mesoscopic and nanoscale systems. In a beautiful experiment, Schwab et al. [1] observed a quantization of the thermal conductance in freely suspended one-dimensional dielectric wires, corresponding to a conductance given by $\pi k_{\mathrm{B}}^{2} T / 6 \hbar$ per transmitted vibrational mode, analogous to the well-known electrical conductance quantization in units of $e^{2} / 2 \pi \hbar$ per spinresolved channel in one-dimensional mesoscopic conductors [2]. The thermal conductance quantization can be understood [3 [5 by using a thermal analog of the Landauer-Büttiker formula 2].

In this paper we consider mesoscopic thermal transport through a wire or weak link in the limit where the phonon transmission probability through the wire is small (the so-called nonadiabatic regime) and the thermal conductance is much less than $\pi k_{\mathrm{B}}^{2} T / 6 \hbar$, the value corresponding to one fully propagating channel. The geometry we consider is shown schematically in Fig. 固.

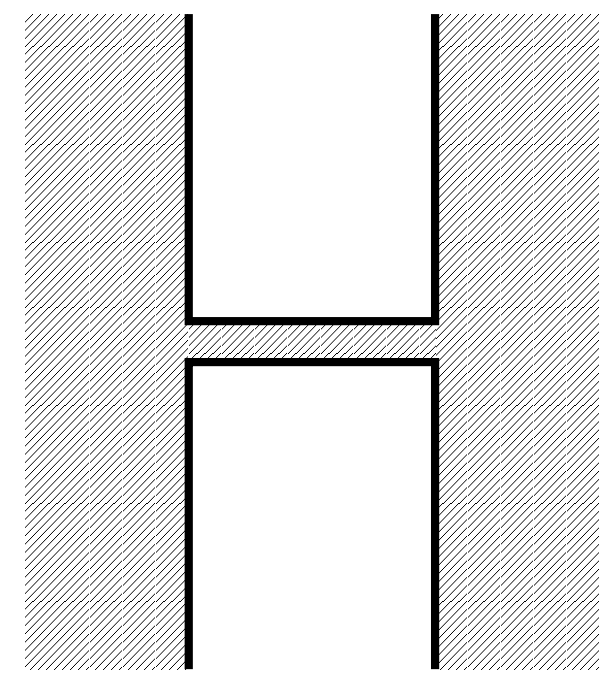

FIG. 1. Mesoscopic wire connected nonadiabatically to bulk dielectrics.
The mechanical bridge may consist of one or more chemical bonds, or by a narrow wire of dielectric material, both of which we model by a harmonic spring of stiffness $K[6]$. We obtain a general expression for the thermal current that can be regarded as a thermal analog of the well-known formula, derived by Schrieffer et al. [7.,8], for the electrical current through a tunneling barrier. Our result can also be interpreted as an application of the thermal Landauer formula in the weak-tunneling limit, with the energy-dependent phonon transmission probability calculated microscopically.

\section{THERMAL CURRENT IN THE WEAK-TUNNELING LIMIT}

We consider two macroscopic dielectrics, $\mathrm{L}$ and $\mathrm{R}$, held at fixed temperatures $T_{\mathrm{L}}$ and $T_{\mathrm{R}}$. The Hamiltonian of the isolated solids is $H_{0}=H_{\mathrm{L}}+H_{\mathrm{R}}$, where

$$
H_{I} \equiv \sum_{n} \omega_{I n} a_{I n}^{\dagger} a_{I n}, \quad I=\mathrm{L}, \mathrm{R} .
$$

The $a_{n I}^{\dagger}$ and $a_{n I}$ are phonon creation and annihilation operators for the left and right sides. The vibrational modes of the isolated bodies are labeled by $n$ and have energies $\omega_{I n}$.

The two bodies are connected by a harmonic spring with stiffness $K$,

$$
\delta H=\frac{1}{2} K\left(u_{\mathrm{L}}^{z}-u_{\mathrm{R}}^{z}\right)^{2} .
$$

Here $u_{I}^{z}$ is the normal component of the displacement field $\mathbf{u}(\mathbf{r})$ at the surface of body $I$ at the point of connection to the weak link, with the local surface normal taken to be in the $z$ direction. Our mesoscopic weak-link model is illustrated in Fig. 2.

The system is described by the Hamiltonian $H=$ $H_{0}+\delta H$. The thermal current is calculated by defining a thermal current operator $\hat{I}_{\text {th }}$ according to

$$
\hat{I}_{\mathrm{th}} \equiv \partial_{t} H_{\mathrm{R}}=i\left[H, H_{\mathrm{R}}\right]
$$


The expectation value of $\hat{I}_{\text {th }}$ is the energy per unit time flowing from the left to the right body. To leading order in $\delta H$ (the weak-coupling limit) we obtain [9]

$$
I_{\mathrm{th}}=\frac{2 \pi K^{2}}{\hbar} \int_{0}^{\infty} d \epsilon \epsilon N_{\mathrm{L}}(\epsilon) N_{\mathrm{R}}(\epsilon)\left[n_{\mathrm{L}}(\epsilon)-n_{\mathrm{R}}(\epsilon)\right],
$$

an expression analogous to the formula derived by Schrieffer et al. [7,8] for the electrical current through a tunneling barrier. Here $n_{\mathrm{L}}(\epsilon)$ and $n_{\mathrm{R}}(\epsilon)$ are Bose distribution functions with temperatures $T_{\mathrm{L}}$ and $T_{\mathrm{R}}$, and

$$
N_{I}(\omega) \equiv-\frac{1}{\pi} \operatorname{Im} D_{I}(\omega)
$$

is the local spectral density, defined in terms of the Fourier transform of the retarded correlation functions

$$
D_{I}(t) \equiv-i \theta(t)\left\langle\left[u_{I}^{z}(t), u_{I}^{z}(0)\right]\right\rangle_{0}
$$

for the isolated macroscopic bodies $\mathrm{L}$ and $\mathrm{R}$. The spectral density defined in (5) is different than the ordinary thermodynamic DOS, even in a homogeneous system. The quantity (5), however, is the one relevant here.

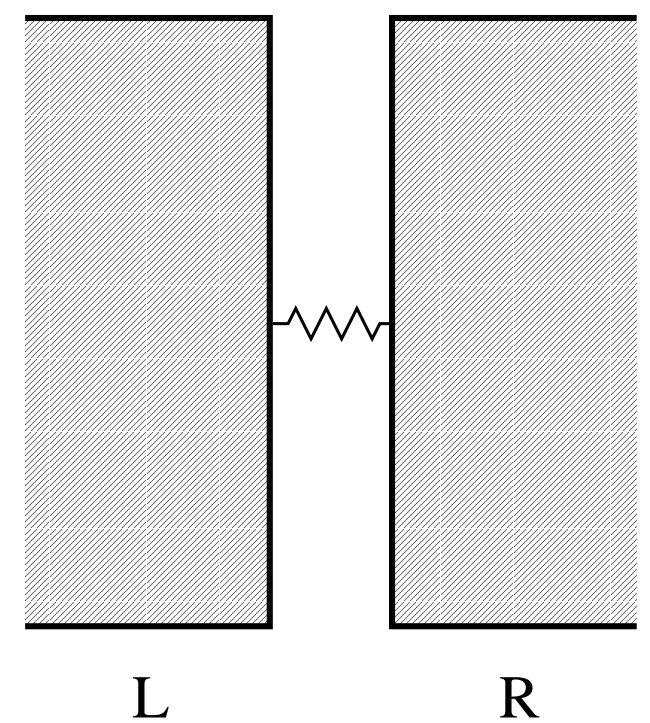

FIG. 2. Our weak link model. Two macroscopic dielectrics are joined by a harmonic spring.

Our result (4) shows that the (linear) thermal conductance of a weak mesoscopic link, defined by

$$
G_{\mathrm{th}} \equiv \lim _{T_{\mathrm{L}} \rightarrow T_{\mathrm{R}}} \frac{I_{\mathrm{th}}}{T_{\mathrm{L}}-T_{\mathrm{R}}}
$$

will vary at low temperatures as a power-law in $T$. If we let $\alpha$ be the exponent characterizing the power-law phonon spectral density of the reservoirs at low energies, then

$$
G_{\text {th }} \propto T^{2 \alpha+1} .
$$

For example, $\alpha=1$ at the planar surface of a semi-infinite isotropic elastic continuum.

A simple application of our theory to a nanostructure consisting of a cylindrical neck of Si material connecting two semi-infinite Si crystals has been given in Ref. [9].

\section{DISCUSSION}

The classical theory of thermal conduction, based on the heat equation and on the concept of a local thermal conductivity, is entirely inapplicable to mesoscopic dielectrics. In a mesoscopic system, thermal resistance is caused by elastic scattering of phonons, whereas in an infinite, disorder-free crystal it is caused by anharmonicity. In the non-mesoscopic regime (for example, at higher temperatures), the thermal conductivity of a narrow bridge is determined by the bridge material's bulk thermal conductivity $\kappa$, but in the mesoscopic regime it is determined by the mechanical properties of the bridge material, through the stiffness $K$.

\section{ACKNOWLEDGMENTS}

This work was supported by NSF CAREER Grant No. DMR-0093217, and by a Research Innovation Award and Cottrell Scholars Award from the Research Corporation. It is a pleasure to thank Andrew Cleland, Steve Lewis, and Aleksandar Milosevic for useful discussions.

[1] K. Schwab, E. A. Henriksen, J. M. Worlock, and M. L. Roukes, Nature 404, 974 (2000).

[2] For a review see C. W. J. Beenakker and H. van Houten in Solid State Physics: Advances in Research and Applications, edited by H. Ehrenreich and D. Turnbull (Academic Press, San Diego, 1991), Vol. 44.

[3] L. G. C. Rego and G. Kirczenow, Phys. Rev. Lett. 81, 232 (1998).

[4] D. E. Angelescu, M. C. Cross, and M. L. Roukes, Superlattices Microstruct. 23, 673 (1998).

[5] M. P. Blencowe, Phys. Rev. B 59, 4992 (1999).

[6] This model assumes that the forces between the two reservoir surfaces, transferred by the link, are mostly longitudinal.

[7] J. R. Schrieffer, D. J. Scalapino, and J. W. Wilkins, Phys. Rev. Lett. 10, 336 (1963).

[8] See also G. D. Mahan, Many-Particle Physics, 3rd ed. (Plenum Publishers, New York, 2000).

[9] K. R. Patton and M. R. Geller, Phys. Rev. B (in press). 